\title{
Analysis and downscaling multi-model seasonal forecasts in Peru using self-organizing maps
}

\author{
By J. M. GUTIÉRREZ ${ }^{1 *}$, R. CANO ${ }^{2}$, A. S. COFIÑO ${ }^{1}$ and C. SORDO ${ }^{2},{ }^{1}$ Department of Applied \\ Mathematics and Computer Science. University of Cantabria, 39005, Santander, Spain; ${ }^{2}$ Instituto Nacional de \\ Meteorología (INM), CMT/CAS, 39005, Santander, Spain
}

(Manuscript received 18 April 2004; in final form 5 January 2005)

\begin{abstract}
We present an application of self-organizing maps (SOMs) for analysing multi-model ensemble seasonal forecasts from the DEMETER project in the tropical area of Northern Peru. The SOM is an automatic data-mining clustering technique, which allows us to summarize a high-dimensional data space in terms of a set of reference vectors (cluster centroids). Moreover, it has outstanding analysis and visualization properties, because the reference vectors can be projected into a two-dimensional lattice, preserving their high-dimensional topology.

In the first part of the paper, the SOM is applied to analyse both atmospheric patterns over Peru and local precipitation observations at two nearby stations. First, the method is applied to cluster the ERA40 reanalysis patterns on the area of study (Northern Peru), obtaining a two-dimensional lattice which represents the climatology. Then, each particular phenomenon or event (e.g. El Niño or La Niña) is shown to define a probability density function (PDF) on the lattice, which represents its characteristic 'location' within the climatology. On the other hand, the climatological lattice is also used to represent the local precipitation regime associated with a given station. For instance, we show that the precipitation regime is strongly associated with El Niño events for one station, whereas it is more uniform for the other.

The second part of the paper is devoted to downscaling seasonal ensemble forecasts from the multi-model DEMETER ensemble to local stations. To this aim, the PDF generated on the lattice by the patterns predicted for a particular season is combined with the local precipitation lattice for a given station. Thus, a probabilistic or numeric local forecast is easily obtained from the resulting PDF. Moreover, a measure of predictability for the downscaled forecast can be computed in terms of the entropy of the ensemble PDF. We present some evidence that accurate local predictions for accumulated seasonal precipitation can be obtained some months in advance for strong El Niño episodes. Finally, we compare the multi-model ensemble with single-model ensembles, and show that the best results correspond to different models for different years; however, the best global performance over the whole period corresponds to the multi-model ensemble.
\end{abstract}

\section{Introduction}

The advances made in ensemble techniques during the last decade have led to a great improvement of seasonal forecast. Nowadays, skilful predictions can be obtained in the tropics some months in advance during El Niño episodes using ensembles from global circulation models (Anderson et al., 2003). Potential end-users of these predictions from different sectors (agriculture, hidrology, etc.) require high-resolution forecasts of surface variables, such as precipitation, to feed their models. However, the spatial resolution of seasonal global models is low $(\approx 100 \mathrm{~km})$ and therefore they are not suitable for soil variables. Downscaling techniques allow us to adapt the low-resolution gridded forecasts to a high-resolution regional grid, or a network of stations, in an

${ }^{*}$ Corresponding author.

e-mail: manuel.gutierrez@unican.es area of interest. In this paper, we analyse this problem considering the seasonal forecasts provided by the multi-model ensemble DEMETER project. This project involves seven global coupled atmosphere-ocean models with different perturbation schemes and formulation, which have been integrated for the period 19572000. Thus, the project provides an appropriate framework for assessing the current skill of seasonal forecast, comparing singlemodel integrations with the multi-model approach, and also with different downscaling techniques (see Palmer et al., 2004, and also http://www.ecmwf.int/research/demeter/).

Due to the huge amount of data given by DEMETER, efficient analysis tools are required to extract useful features, providing more simple and manageable information. Data mining techniques are advanced statistical methods developed for this task (see, for example, Han and Kamber, 2000). The selforganizing map (SOM) is one of the most popular data mining techniques for clustering high-dimensional data, because it has 
prominent visualization properties (see Cavazos, 1999; Oja and Kaski, 1999; Hewitson and Crane, 2002, for some applications). In the first part of the paper, we apply this technique for clustering and analysing a space of daily atmospheric patterns over Peru, characterizing the climatology. In particular, we consider daily analysis data from the ERA40 reanalysis project during the period 1979-1998 (the period corresponding to the available DEMETER data for this study). The SOM provides both a partition in clusters (represented by the centroids or reference vectors) and a projection of the resulting reference vectors into a low-dimensional lattice (usually a regular two-dimensional lattice) which preserves the neighbourhood constraints of the highdimensional data space. Thus, the resulting lattice represents the climatology, and is appropriate for displaying and characterizing particular events (e.g. El Niño episodes) by means of the distributions drawn in the lattice by the associated patterns. For instance, the probability density functions (PDFs) defined in the lattice by El Niño episodes are restricted to particular regions which characterize this phenomenon. In this paper, we show that the lattice can also be used to represent the seasonal ensemble forecasts given by DEMETER models (including the multi-model ensemble). We also show that the similarity between an ensemble prediction and the climatology (the anomaly of the forecast), or between the predictions of different models, can be quantitatively measured using the relative entropy of the corresponding PDFs. Thus, this paper extends the work by Eckert et al. (1996), who introduced the entropy for characterizing the dispersion, or spread, of the ensemble in a similar framework (medium-range ensemble prediction systems).

In the second part of the paper, the SOM is used for downscaling seasonal ensemble precipitation forecasts. Different downscaling techniques (analogue methods and weather generators) have been applied to DEMETER data in mid-latitudes, and the results are also presented in this volume (see, for example, Díez et al., 2005; Feddersen and Andersen, 2005). However, the skill of seasonal models is larger in the tropics, and thus encouraging results could be obtained in this area. We present an application of the SOM to this problem, extending a recently proposed method (Gutiérrez et al. 2004) which introduces an analogue-based downscaling method using clustering techniques (see Wilby and Wigley, 1997; Zorita and von Storch, 1999, for a more general introduction to analogue-based downscaling techniques). A forecast for a local station is obtained relating the atmospheric patterns for each particular cluster to local observations of daily precipitation. Then, the PDF corresponding to the seasonal forecast is converted to precipitation amounts. The graphical visualization capabilities of the SOM make the whole process intuitive and visually appealing. This technique is applied to Northern Peru, obtaining promising results during the strong El Niño periods of 1982/1983 and 1997/1998 (with lead times ranging from one to six months).

This paper is organized as follows. In Section 1 we give an introduction to the problem. In Section 2 we describe the data and models used in this work. In Section 3 we present a brief introduction to SOMs, and in Section 3.1 we illustrate their application to characterize different events by means of PDFs. A quantitative characterization of the PDFs is presented in Section 3.2, using the concept of entropy. The analysis of DEMETER data with this technique is presented in Section 4. In Section 5 we introduce a downscaling method based on the above ideas, and in Section 5.1 we present some results. Finally, some conclusions are given in Section 6 .

\section{Data and area of study}

We analyse seasonal precipitation in the tropical area of Nothern Peru considering two nearby stations: Sausal de Culucán and Morropón (see Fig. 1). This region of Peru is the most influenced during El Niño episodes, the occurrences of which are illustrated in Fig. 2a; this figure shows the monthly values of

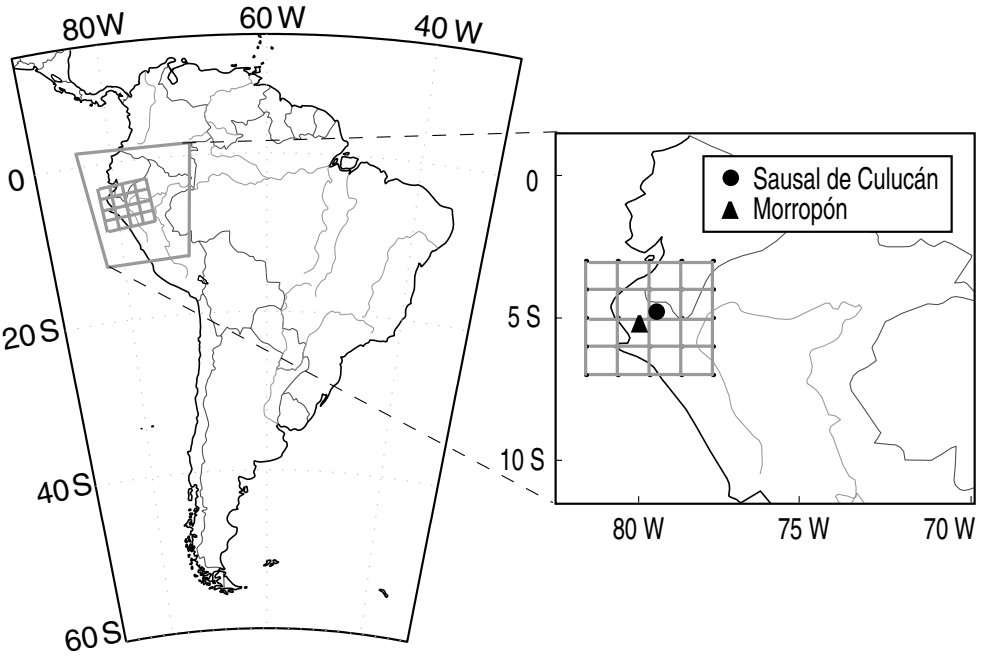

Fig. 1. Area of study in the north of Peru showing the grid used to define the atmospheric patterns and the location of the two stations used in the analysis. 

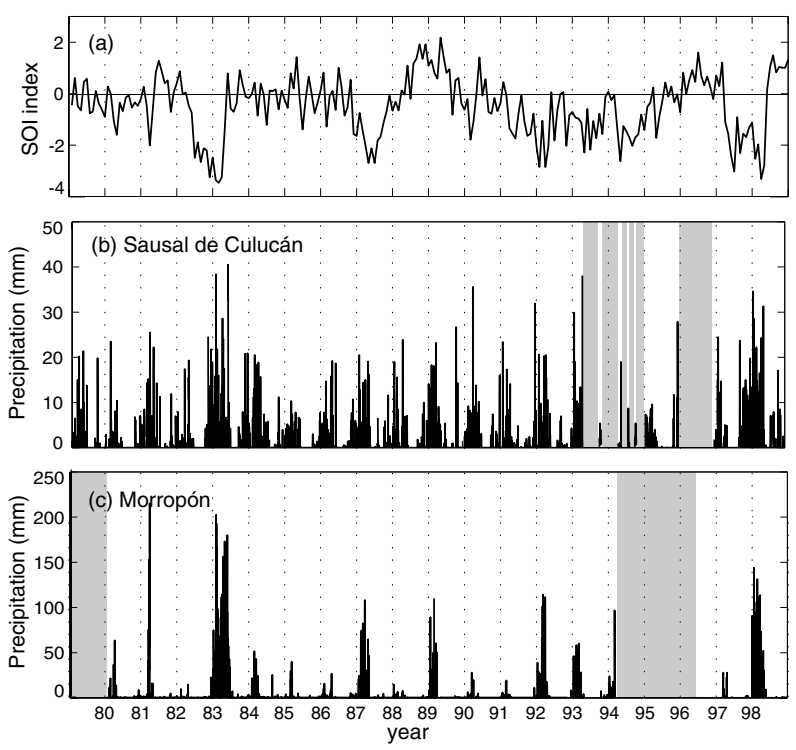

Fig. 2. (a) Monthly Southern Oscillation Index (SOI) corresponding to the period 1980-1998. Daily accumulated precipitation in (b) Sausal de Culucán and (c) Morropón for the same period. Shaded areas indicate missing data periods.

the Southern Oscillation Index, obtained as the Tahiti-Darwin sea level pressure difference (see Allan et al., 1991, for more details).

Figure 2 also shows the daily accumulated precipitation series for the period 1980-1998 observed at the above two stations. This figure illustrates the different precipitation regimes exhibited by these stations. On the one hand, Sausal de Culucán shows a uniform precipitation regime (Fig. 2b) with maximum precipitation values attained during El Niño periods; this station exhibits a marked annual precipitation cycle, with maxima during the period December-March. On the other hand, the precipitation regime at Morropón (Fig. 2c) is strongly related to El Niño events, reaching accumulated monthly precipitation values over $1000 \mathrm{~mm}$ during some severe episodes; in this case, the most rainy season corresponds to December-February (DJF). Shaded areas in Fig. 2 indicate missing data periods (e.g. from 1994 to 1996 for Morropón). The climatology for each of the stations is defined using the available data. Moreover, the SOM technique used in this paper is robust to this problem and the learning algorithms automatically take care of missing data during the training process.

In this paper we consider the period DJF to analyse seasonal precipitation predictability, because it corresponds to the most rainy season in the area of interest. The precipitation regime of Morropón makes standard seasonal prediction based on terciles (dry/normal/wet) useless, because no discrimination between strong and moderate El Niño periods is achieved with this ternary division. Therefore, in this paper we focus on the problem of forecasting a seasonal precipitation amount for a local station using the information provided by one, or several, numerical seasonal atmospheric models (seasonal downscaling).

In order to characterize a representative pattern for the daily atmospheric configuration, we have considered a local area grid constrained to the region of interest (see Fig. 1). Note that other alternatives are possible (global synoptic patterns, etc.), but dynamical local area patterns have recently been shown to be appropriate in a similar study (Gutiérrez et al., 2004). For each grid point we have considered geopotential, temperature, specific humidity and $U, V$ wind components, at 00 and 24 UTC (similar to the observation window) for 850 and $500 \mathrm{hPa}$, to define the atmospheric pattern with a total dimension of $500=25$ (grid points) $\times$ 5 (variables) $\times 2$ (levels) $\times 2$ (hours). Thus, in this paper we use the atmospheric data given by $\mathbf{x}_{k}=\left(x_{k 1}, \ldots, x_{k 500}\right), k=$ $1, \ldots, N$, where $N$ is the total number of available daily patterns. The above data are generic and can be optimized by analysing the most influencing variables for each phenomenon of interest (for instance, the 500-hPa geopotential could be removed from the list, due to its low variance at tropical latitudes). However, data mining techniques such as SOMs have the capability for automatically discovering the most influencing features among the above huge amount of data (see Cofiño et al., 2003, for more details about data mining properties of SOMs). Thus, instead of studying the optimal choice of variables for this problem, we consider a pattern with many relevant items of information and let the SOM work to discover important features among the data. Then, we analyse the resulting clusters looking for a physical interpretation.

In this paper we use the output of four DEMETER models: the European Centre for Medium-Range Weather Forecasts (ECMWF); the Centre National de Recherches Météorologiques (CNRM); the UK Meteorological Office (UKMO); Max-PlanckInstitut für Meteorologie (MPI). The remaining models were not available for the period of analysis 1979-1998 when this work was done. These models run from sets of initial conditions, each slightly different from one another, but consistent with available observations. For each single model, nine-member ensemble integrations ran four times per year (1 February, 1 May, 1 August and 1 November, respectively) over six-month periods, providing daily forecasts. Thus, for a given season (e.g. DJF), each of the models provides $90 \times 9=810$ daily patterns. Note that each daily pattern of a seasonal forecast is not representative of the corresponding day, but the only information provided by the forecast is given in terms of the predicted anomalies, obtained by comparison of the global daily data with the corresponding seasonal climatology.

Besides the DEMETER seasonal forecasts, we have considered the analysis fields of the ERA40 reanalysis to characterize daily configurations for the same period considered for the DEMETER data. In this case, because all patterns are analysis directly obtained from the assimilation process, they define the corresponding atmospheric configuration of the day, and can be related to the simultaneous local observations. 
This huge amount of data requires efficient analysis and downscaling algorithms to be efficiently processed. Data mining techniques such as SOMs have been designed for this task.

\section{Self-organizing maps for data analysis}

In the last decade, several statistical methods have been developed in the field of data mining to deal with huge amounts of information in an efficient form (see Cofiño et al., 2003, for some applications in meteorology). These techniques work by extracting features from data, giving a more compact and manageable representation of some important properties contained in the data. Standard methods in data mining include clustering techniques (for obtaining a set of reference vectors representing the data), dependency graphs (for representing dependencies among the variables), association rules, etc. The SOM is one of the most popular clustering techniques in this field. A SOM summarizes the high-dimensional data space in terms of a set of reference vectors (cluster centroids) having a spatial organization corresponding to a low-dimensional lattice (usually a two-dimensional regular lattice). This is done by a topology preserving projection from the original data space into the low-dimensional lattice. Thus, as we shall see later, the lattice provides a natural support for projecting distributions from the high-dimensional space, so they are transformed into PDFs on the lattice.

Consider the high-dimensional data space given by the atmospheric patterns described in Section 2 for the ERA period. A $\mathrm{SOM}$ is formed by an arbitrary number of clusters $C_{1}, \ldots, C_{m}$ located on a two-dimensional lattice for visualization purposes. Each cluster $C_{k}$ is associated with two vectors. On the one hand, the vector $\mathbf{c}_{k}=\left(i_{k}, j_{k}\right)$ describes the position of the cluster on the lattice (for instance, the $3 \times 3$ lattice shown in Fig. 3). On the other hand, the reference vector $\mathbf{v}_{\mathrm{k}}=\left(v_{k 1}, \ldots, v_{k \mathrm{n}}\right)$ represents the position of the cluster centroid in the data space. In the original work of Kohonen (1995), a SOM was introduced following a neural network analogy, where $C_{k}$ was associated with a neuron (or processing unit) connected to each of the components of the data space through a weight vector $\mathbf{v}_{\mathrm{k}}$ (see Fig. 3).

The kernel of the SOM is a learning method to adjust the reference vectors to data using an unsupervised algorithm (no domain knowledge is needed and no human intervention is required), so that the resulting clusters represent meaningful subgroups within the data (weather classes). The goal of the algorithm is to minimize an overall within-cluster distance $\mathrm{d}\left(C_{k}\right)$ from the data vectors $\mathbf{x}_{i}$ within the cluster to the corresponding reference vector $\mathbf{v}_{k}$, for each cluster $C_{k}$ :

$\sum_{k=1, \ldots, m} \mathrm{~d}\left(C_{k}\right)=\sum_{k=1, \ldots, m} \sum_{\mathbf{x}_{\mathbf{i}} \in C_{k}}\left\|\mathbf{x}_{\mathbf{i}}-\mathbf{v}_{\mathbf{k}}\right\|^{2}$

The algorithm runs iteratively, adapting the reference vectors smoothly to minimize eq. (1). This algorithm is similar to standard iterative clustering algorithms, such as the $k$-means method

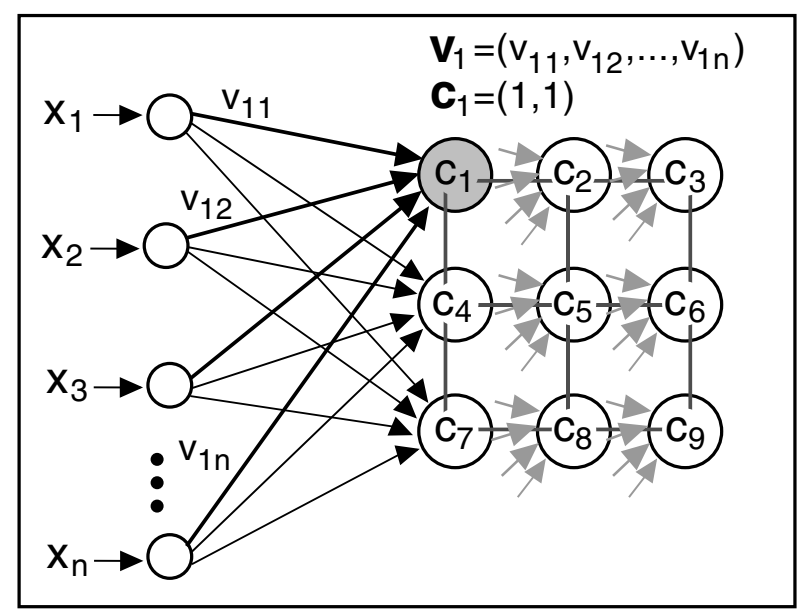

Fig. 3. Schematic diagram of a $3 \times 3$ two-dimensional SOM where each cluster (or neuron) $C_{k}$ has associated a lattice-position vector $\mathbf{c}_{k}=$ $\left(i_{k}, j_{k}\right)$ and a reference vector (or weight) $\mathbf{v}_{k}=\left(v_{k 1}, \ldots, v_{k \mathrm{n}}\right)$.

(see Gutiérrez et al., 2004, for more details), which starts from a random initialization of the reference vectors (see Peña et al., 1999, for a discussion of different initialization procedures). Then, the training proceeds in cycles. On each training cycle, each of the data vectors $\mathbf{x}_{i}$ is considered and the best-matching (or 'winning') reference vector $\mathbf{v}_{w(i)}$ is obtained as the one closest to the data vector:

$\left\|\mathbf{v}_{w(i)}-\mathbf{x}_{i}\right\|=\min _{k}\left\{\left\|\mathbf{v}_{k}-\mathbf{x}_{i}\right\|, k=1, \ldots, m\right\}$.

Here, $1 \leq w(i) \leq m$ denotes the index of the winner reference vector, which is moved towards the data vector in the following form:

$\mathbf{v}_{w(i)}=\mathbf{v}_{w(i)}+\alpha\left[\mathbf{x}_{i}-\mathbf{v}_{w(i)}\right]$.

$0<\alpha<1$ is the learning rate and controls the 'velocity' of the learning process (a small value of this parameter leads to a smooth and slow learning process, whereas a high value leads to a fast but unstable learning process). The batch version of the algorithm accumulates the increments from each data vector and updates the reference vectors after a whole cycle.

What makes the SOM different from standard iterative clustering algorithms is that the training process includes a neighbourhood adaptation mechanism so neighbouring clusters of the winning reference vector in the two-dimensional lattice space are also adapted towards the sample vector, thus projecting the topological neighbourhood relationships of the high-dimensional data space on to the lattice. The training algorithm of the SOM is based in the following equation

$\mathbf{v}_{k}=\mathbf{v}_{k}+\alpha h[w(i), k]\left(\mathbf{x}_{i}-\mathbf{v}_{k}\right), k=1, \ldots, m$,

which is used in the above iterative process instead of the generic iterative clustering adaptation eq. (3). The function $h(j, k)$ is a 
neighbourhood kernel on the SOM lattice, centred on the vector $\mathbf{c}_{j}$, which determines the rate of change around the winner unit (usually a Gaussian function is considered):

$h(j, k)=\exp \left(-\frac{\left\|\mathbf{c}_{j}-\mathbf{c}_{k}\right\|}{\sigma}\right)$.

As a consequence of the neighbourhood kernel, during the iterative training the SOM behaves like a flexible lattice folding on to the cloud formed by the data in the original $n$-dimensional space. Both the learning rate $\alpha$ and neighbourhood kernel amplitude $\sigma$ decrease monotonically with time, softening the folding process; a linear decay to zero is usually chosen for these functions. For a detailed description of different implementations of the method, the reader is referred to Oja and Kaski (1999) and Hewitson and Crane (2002).

SOMs have recently been applied to some problems related to the topic of this paper (see Hewitson and Crane, 2002, for a reader friendly introduction to SOMs in climatolgy). On the one hand, Cavazos $(1999,2000)$ applies SOMs from a climatic perspective to classify circulation fields and derive relationships with daily precipitation at local scale (the National Center for Environmental Prediction/National Center for Atmospheric Research reanalysis is used in this case); however, no application to operative short-range or ensemble forecast is performed. On the other hand, Eckert et al. (1996) apply SOMs to characterize and group the ensemble members of a medium-range ensemble forecast; however, in this case, no connection is made with local forecast (downscaling). The present paper shares a common methodology with the above described works, but applies it to a different framework, providing both operative local seasonal forecasts from a multi-model ensemble (downscaling) and an estimation of the predictability of the resulting forecasts (ensemble analysis).

Moreover, the number of clusters considered in the above works is only suitable for the classification of global circulation from a climatic perspective, and it does not give enough discriminant power for the purpose of the present study. For this reason we have considered SOM lattices of $8 \times 8=64$ groups (an average of $\approx 100$ ERA days for each cluster for the period 1979-1998) following the indications of Gutiérrez et al. (2004). The reference vectors were initialized considering a regular two-dimensional lattice over the space spanned by the first two principal components of the data (see Peña et al., 1999, for more details about initialization procedures). We used the MeteoLab toolbox for Matlab (http://grupos.unican.es/ai/meteo/MeteoLab.html) for training different SOMs with different configurations of parameters, using a linear decay to zero for both the learning rate and neighbourhood amplitude after 5000 cycles. Figure 4 shows two of the fields (temperature and specific humidity at $850 \mathrm{hPa}$ ) corresponding to the resulting reference vectors for each of the $8 \times 8$ clusters, as displayed in the lattice (the rest of the fields have been omitted from this figure to avoid visual confusion).
From this figure we can see how close reference fields in the lattice exhibit similar patterns, whereas more distant fields become increasingly different.

As we shall see later, the bottom-left corner of the resulting lattice is associated with patterns related to El Niño events, whereas the top-right corner is associated with the La Niña phenomenon. Figure 5 shows the fields associated with an El Niño cluster the one with lattice position $c=(7,1)$. This figure displays the complete atmospheric information corresponding to the reference vector: specific humidity, temperature and geopotential for 850 and $500 \mathrm{hPa}$, as well as the corresponding wind fields. From this figure we can see the average effect given by the reference vector (or centroid) of a group of patterns associated with El Niño events. Trade winds and Peruvian current drive the main atmosphere-ocean state in this area. When the trade winds are blocked, a flux of warm water coming from the north extends an extremely warm and wet tongue (El Niño current) which meets the cold Peruvian current just in front of the west coast of Northern Peru (see Figs. 5a and c); this convergence of airmasses in the nearby of the big Andes ridge is very effective to produce the deep convection that produces heavy precipitation.

Different SOMs were trained obtaining similar results, but with different relative positions of the patterns in the lattice.

\subsection{Characterizing distributions of seasons and events}

The topology preservation property of the SOM is very convenient for characterizing distributions of events over the reanalysis period. A subset of patterns (e.g. DJF season) of the reanalysis data base defines a PDF on the regular lattice, according to the histogram of winner clusters for each pattern. Due to the topology preserving property of the SOM, there is an equivalence between the variance of the resulting PDF and the variance of the original subset of atmospheric patterns in the reanalysis space. For instance, Fig. 6 shows the interpolated PDFs corresponding to the histograms generated in the lattice of the SOM shown in Fig. 4 by different periods of ERA40: (a) corresponds to the climatology of the DJF 'season' for the period 1979-1998, (b) and (c) correspond to strong El Niño DJF periods, and (d) and (e) correspond to DJF periods for normal years. From this figure we can see how the variance is larger for the DJF 1979-1998 period, which is distributed in the central lower region of the lattice (note that the lattice has been obtained using the whole 1979-1998 period). On the other hand, the densities of individual years have smaller variance; moreover, the "normal years' resemble the climatology more than El Niño periods, as expected, because 'normal years' are closer to climatology than the anomalous situations corresponding to El Niño years. These conclusions can be easily drawn by visual inspection due to the visually appealing properties of the SOM. This discrimination capability can be numerically characterized in terms of entropies. 


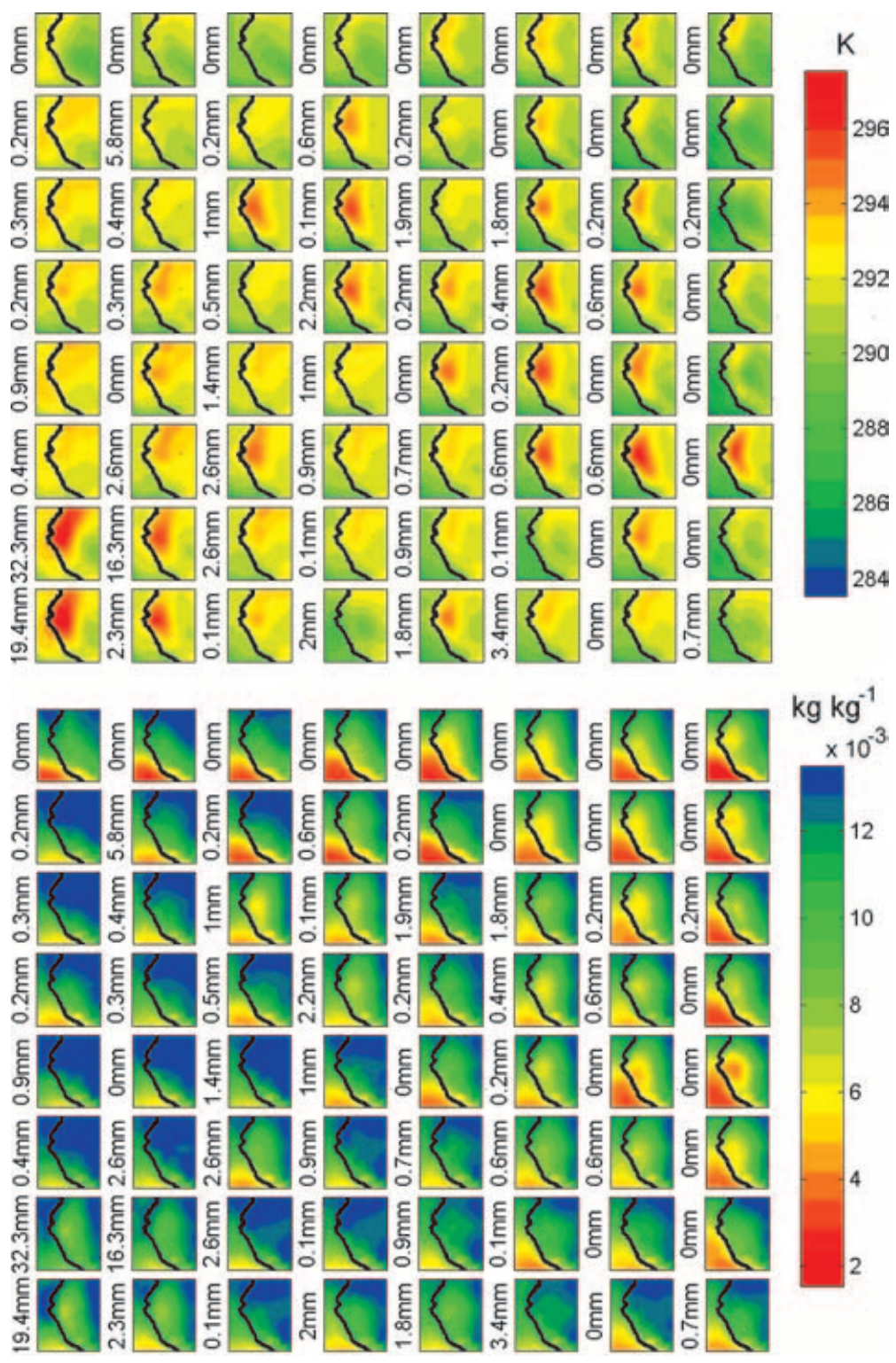

Fig. 4. Temperature fields for the $8 \times 8$ SOM reference vectors at $850 \mathrm{hPa}$ (upper panel) and specific humidity fields at $850 \mathrm{hPa}$ (lower panel). Note that the fields are drawn over an area larger than the local grid shown in Fig. 1; however, the ERA dates associated with each cluster have been obtained using the local area grid and the fields have been enlarged only for the sake of visualization. The cluster index grows from top to bottom and from left to right ( $c_{11}$ is shown in the top-left corner of the figure).

\subsection{Entropy and relative entropy}

Since Shannon (1948), the concept of entropy has been used in many fields to characterize disorder, or spread, of general nonGaussian PDFs. In the previous section we have described the application of SOMs for representing a set of atmospheric patterns by means of the PDF, or histogram, defined on the lattice of a SOM. In particular, we have used an $8 \times 8$ lattice, which yields to a set of probabilities $\left(p_{1}, \ldots, p_{64}\right)=\left[P\left(c_{1}\right), \ldots, P\left(c_{64}\right)\right]$. In this case, the entropy is given by (see Eckert et al., 1996, for more details):

$E(P)=-\sum_{i} p_{i} \log p_{i}$
The maximum value of the entropy is associated with a uniform distribution, whereas a minimum zero value corresponds to a delta-like probability $\left(p_{i}=1\right.$, for some index $i$ ). Note that a uniform distribution on the lattice corresponds to the climatology of the $20 \mathrm{yr}$ used to train the SOM. The label of Fig. 6a shows the entropy associated with the DJF period. Eckert et al., (1996) introduced the entropy in this context to characterize the spread of Ensemble Prediction System (EPS) forecasts.

Analogously, the relative entropy (also called the KullbackLeibler distance between probability functions) measures the degree of similarity/disimilarity between two different PDFs. Thus, we can compare the PDFs corresponding to different events, or years, with the climatological PDF, obtaining a measure of anomaly (in the sense of distance from climatology). Given two 
(a) $\mathrm{H} 850 \mathrm{hPa}$

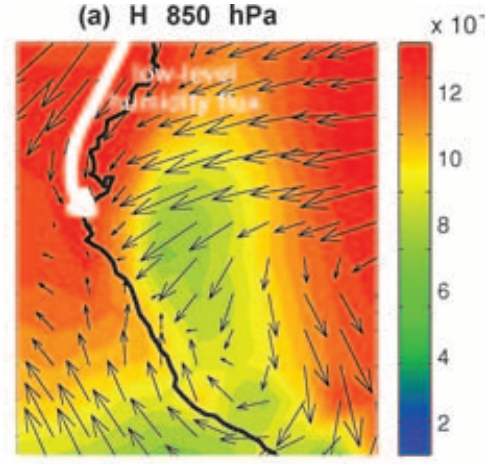

(c) T $850 \mathrm{hPa}$

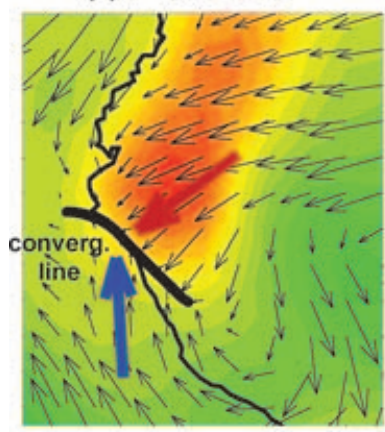

(e) $\mathrm{Z} 850 \mathrm{hPa}$

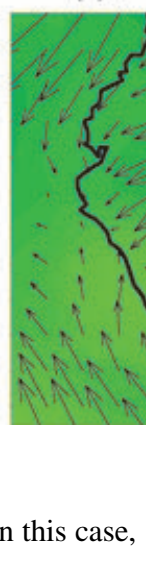
the SOM lattice), the relative entropy is defined as

$\mathrm{RE}(P \mid Q)=\sum_{q_{i} \neq 0} p_{i} \log \frac{p_{i}}{q_{i}}$.

It follows that $\operatorname{RE}(P \mid Q) \geq 0$, and the value zero is only attained when $P \equiv Q \Leftrightarrow p_{i}=q_{i}$ for all $i$. The labels of Figs. 6b-e show the relative entropy of the period DJF for different years compared with the climatological PDF shown in Fig. 6a. Note that the relative entropy of the El Niño periods is significantly larger than that corresponding to 'normal years', indicating a larger dissimilarity with climatology. These results agree with the qualitative conclusions drawn from a visual inspection of the PDFs.

In the following section we analyse the seasonal forecasts given by the DEMETER project. As we shall see, the concept of relative entropy gives us a measure of predictability in this context.

\section{Analysing DEMETER seasonal forecasts}

The study of the previous sections has been performed using reanalysis data. However, we can make a similar analysis using the seasonal forecasts given by DEMETER models. The resulting PDFs give us valuable information about the different models (how they are distributed according to ERA climatology) and the forecasts (spread of the ensembles, anomaly, etc.). Figure 7 shows the PDFs corresponding to the forecasts of ECMWF, UKMO, MPI and CNRM models for the DJF season (1-3 month lead time) for different periods: (b)-(f) 1997/1998 and (g)-(k) 1984/1985, corresponding to a strong El Niño period and a normal period, respectively (in different columns). 
(a) DJF 1979-1998. E=3.69

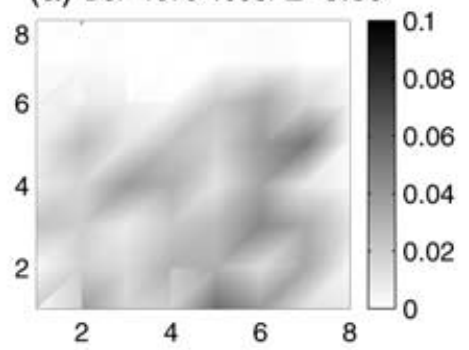

(b) DJF 1982/83. RE=1.77

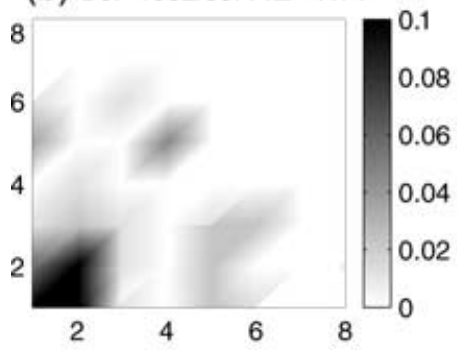

(d) DJF 1979/80. RE $=0.75$

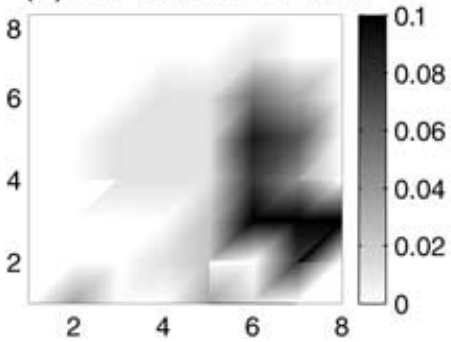

(c) DJF 1997/98. RE=1.51

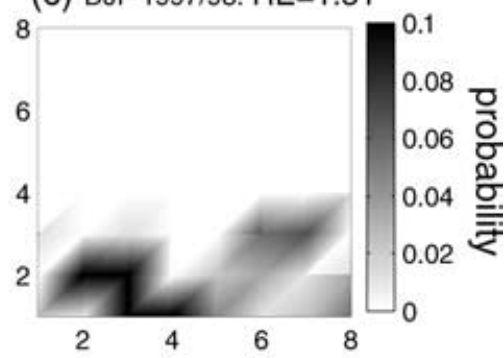

(e) DJF 1984/85. RE $=0.93$

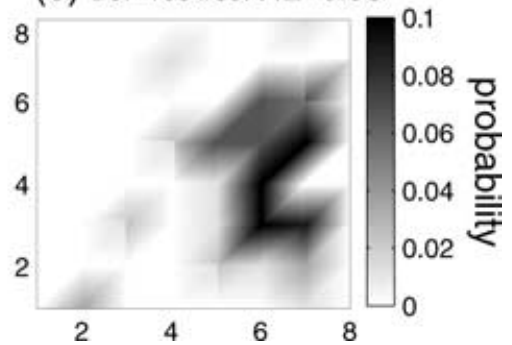

Fig. 6. PDFs over the SOM lattice for different DJF periods: (a) 1979-1998 (climatology), (b) 1982/1983, (c) 1997/1998, (d) $1979 / 1980$ and (e) $1984 / 1985$.
Figures $7 \mathrm{~b}$ and $\mathrm{c}$ show the PDF of the multi-model (DEMETER) obtained by forming a superensemble with the forecast members of all models. In these cases, the PDF is obtained considering the 90 (days) $\times 4$ (models $) \times 9$ (ensemble members $)=3240$ forecasted patterns. Finally, Fig. 7a shows the PDF for the multimodel DJF 1979-1998 period.

As a measure of the similarity, or agreement, of each model with the DEMETER superensemble, the relative entropies of each model versus the multi-model PDF have been computed (see the 'RE' labels of Fig. 7). The MPI model exhibits the largest dissimilarity from the multi-model PDF (Figs. 7f and g); for instance, for the period $1984 / 1985$, the relative entropy of the MPI model, 2.28, is caused by the density spot in the bottomright part of the lattice, associated with atmospheric patterns which have been forecast by no other model (only partially by the ECMWF model) and hence have low representation in the multi-model ensemble.

Figures $6 \mathrm{~b}$ and $\mathrm{c}$ show that strong El Niño events are restricted to a certain area of the lattice. Thus, a skilful seasonal forecast for an El Niño period would also be restricted to this area. The relative entropy of the multi-model PDF versus the ERA 19821998 DJF period (Fig. 6a) is shown in Figs. 7b and g (label 'RE_ERA'). This value is larger for the period 1997/1998, in- dicating the anomaly captured for the models for that year. If we use the climatological PDF of the multi-model DEMETER instead (Fig. 7a), a similar result is obtained, but now the relative entropies 'RE_DEM' are smaller, because we are using the proper climatology as the basis for the relative entropy. These results show that the seasonal DEMETER forecast for El Niño 1997/1998 was quite skilful for 1-3 month lead time.

Figure 8 shows the relative entropy value computed for each separate model and for the multi-model DEMETER (solid line) for the DJF period (1-3 month lead time) from 1982-1998. This curve shows how the years corresponding to strong El Niño events are clearly identified as peaks. Because the relative entropy value identifies how anomalous a forecasted season is, compared with the climatology, it also gives a measure of the value of the seasonal forecast. Relative entropies are computed considering (a) the ERA40 climatology and (b) the DEMETER climatology, and the results are similar in both cases.

In order to check the performance of the models as a function of the lead time, we have also computed the relative entropies for December-January considering 5-6 month lead time forecasts for the periods $1982 / 1983$ and $1997 / 1998$, obtaining the values 1.15 and 2.02, respectively (note that there is no February forecast for August integrations). Thus, whereas the relative entropy 


\section{DEMETER DJF 1979-1998}

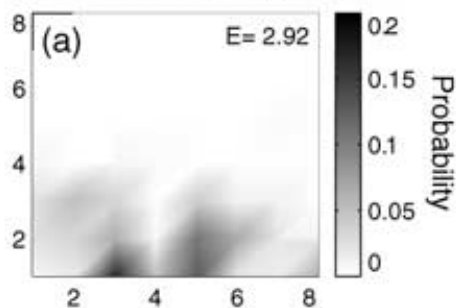

DJF $1997 / 98$
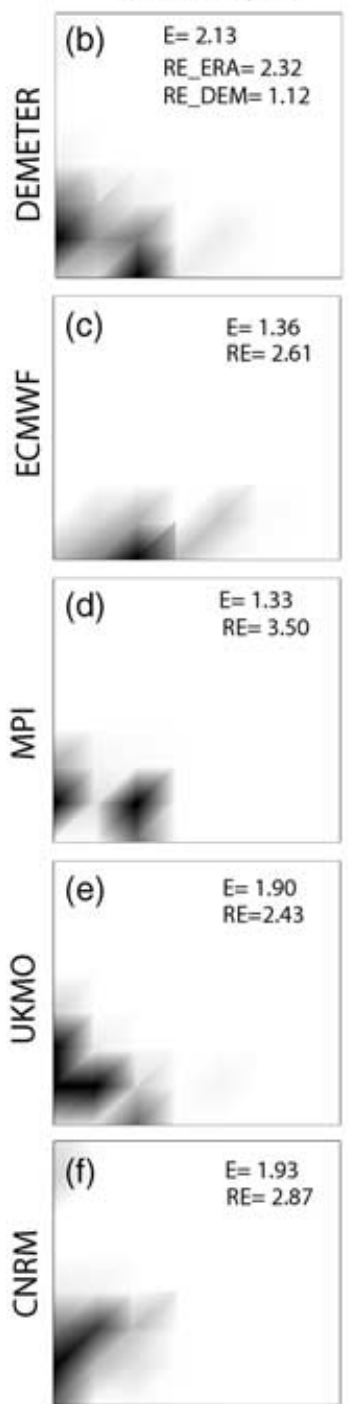

DJF $1984 / 85$
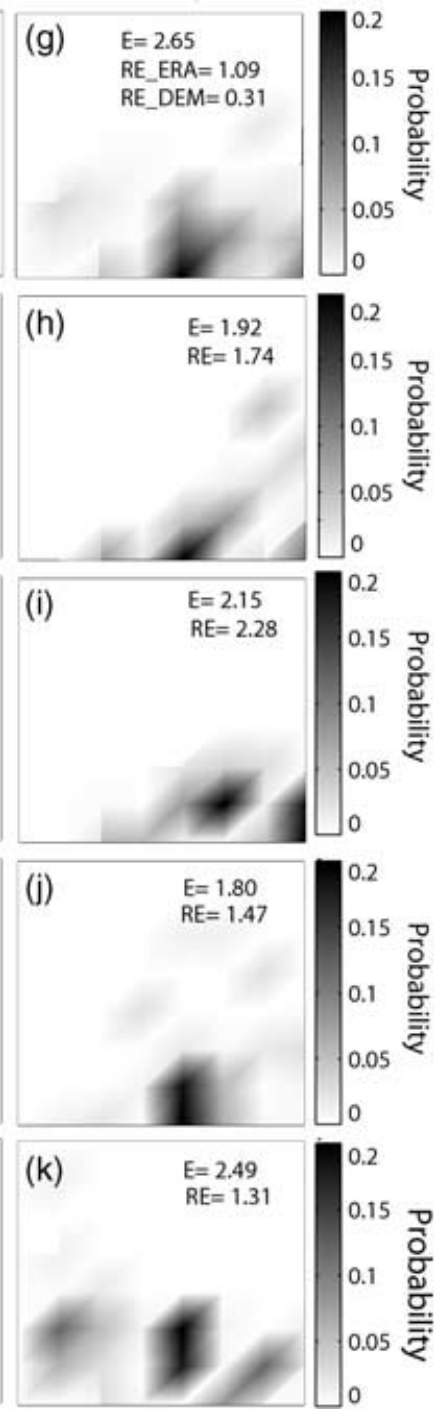

Fig. 7. (a) Climatological PDF of the multi-model DEMETER 1979-1998. (b), (g) PDFs of the multi-model DEMETER for two different years. (c)-(f) and (h)-(k) individual PDFs for each of the models (ECMWF, MPI, UKMO and CNRM) for the periods DJF 1997/1998 and 1984/1985, respectively.

of the period 1982/1983 with 5-6 month lead time decreases to a normal value, the relative entropy for the year 1997/1998 is still high, indicating that the models detected the anomaly at least five months in advance.
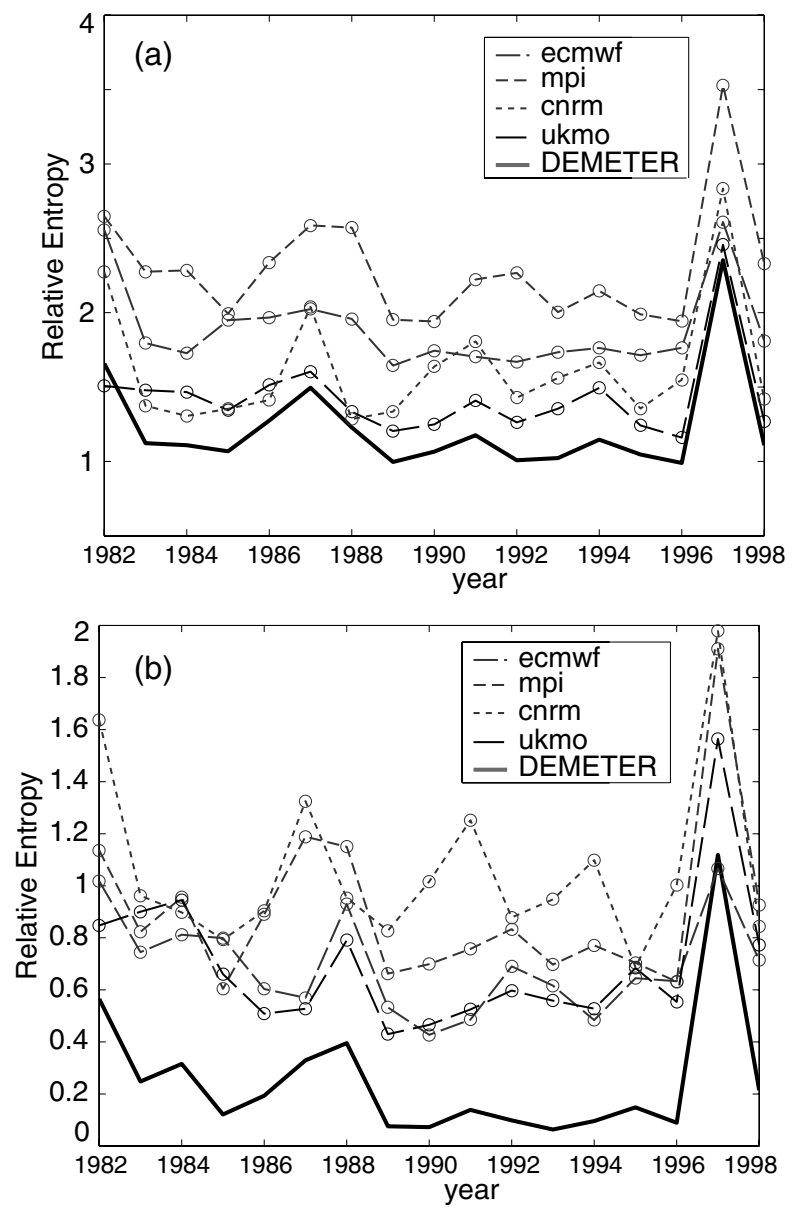

Fig. 8. Relative entropy of the DJF PDFs associated with the multi-model DEMETER and with each individual model for the years 1982-1998 compared with (a) the climatic PDF for ERA and (b) the climatic PDF for DEMETER.

\section{Downscaling method}

The above technique can also be used to increase the spatial resolution of the seasonal forecast by connecting atmospheric fields with series of daily observations at local stations. Figure 1 shows the location of two nearby stations with different precipitation regimes. Figure 2 shows the time series of daily precipitation values available for each of the stations. Each of these series defines a function on the SOM lattice corresponding to the average daily precipitation for the ERA dates corresponding to each of the clusters. Other statistics for estimating the cluster precipitation value have been considered recently (Gutiérrez et al., 2004). However, although non-central estimators (e.g. percentile 75 ) outperform the 'mean' estimation in clusters with extreme events, they could globally overestimate the precipitation value. Thus, we consider the mean value as a global estimator in this paper.

The resulting function characterizes the local climatology of the station conditioned to the atmospheric configurations 

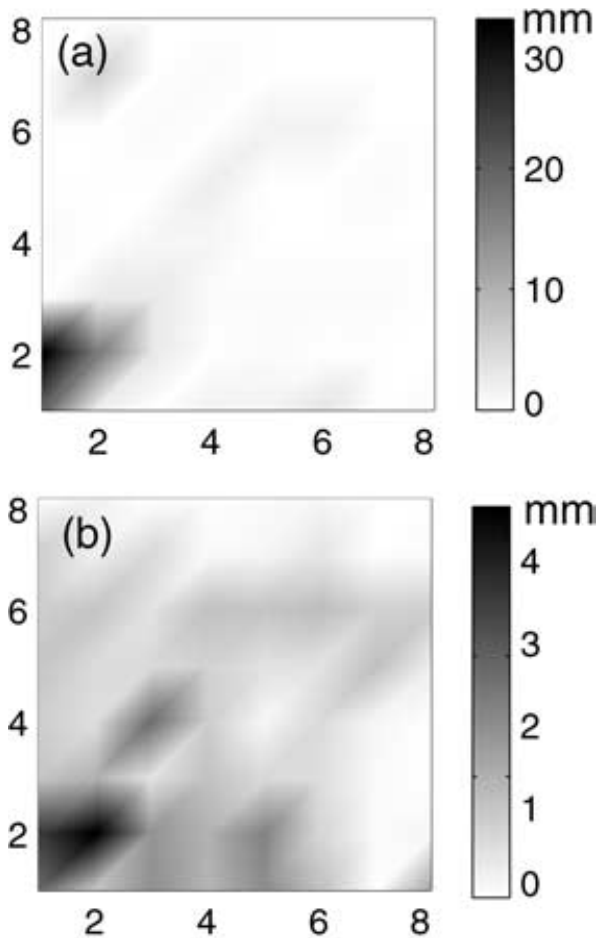

Fig. 9. Mean daily precipitation associated with the atmospheric patterns for each of the clusters of the SOM for (a) Morropón and (b) Sausal de Culucán.

represented by the different clusters (weather classes). For instance, Fig. 9 shows the observation functions defined in the SOM lattice by the observations registered in Morropón and Sausal de Culucán during the period 1979-1998. Note that the precipitation at Morropón is almost constrained to the region of the lattice associated with El Niño; moreover, the maximum daily mean precipitation value reaches $30 \mathrm{~mm}$. On the other hand, precipitation in Sausal de Culucán is more uniform on the lattice and the maximum mean value is below $5 \mathrm{~mm}$. Therefore, the precipitation regime of these two stations is very different and depends on El Niño phenomena in different forms, as already shown in Fig. 2.

Because these functions relate atmospheric patterns (represented by a reference vector) to local observations, they can be combined with the PDF associated with a seasonal forecast in order to obtain a local prediction. This downscaling scheme is similar to the clustering-based analogue technique recently introduced by Gutiérrez et al. (2004). This is an alternative to analogue techniques and works by applying clustering techniques for partitioning the reanalysis data base into meaningful subgroups, each associated with a reference pattern, which represents an specific atmospheric scenario. This process is computationally expensive, but it is performed a single time (in our case, this process is carried out using the SOM learning algorithm). Afterwards, each forecast pattern is assigned to its winner cluster, according to its distance from the reference vectors. Thus, the search of analogues is conducted in a reduced space (the space of reference vectors) reducing significantly the computational cost (see Zorita and von Storch, 1999, for an overview to analogue downscaling methods).

This algorithm can be easily adapted to work with EPS, as shown in Fig. 10. For each season, nine forecast patterns are daily available for each of the four models, defining four different PDFs on the SOM lattice (or a single PDF if the models are mixed together). The local forecast is finally obtained combining these PDFs with the function assigning the mean precipitation to each cluster and each of the stations.

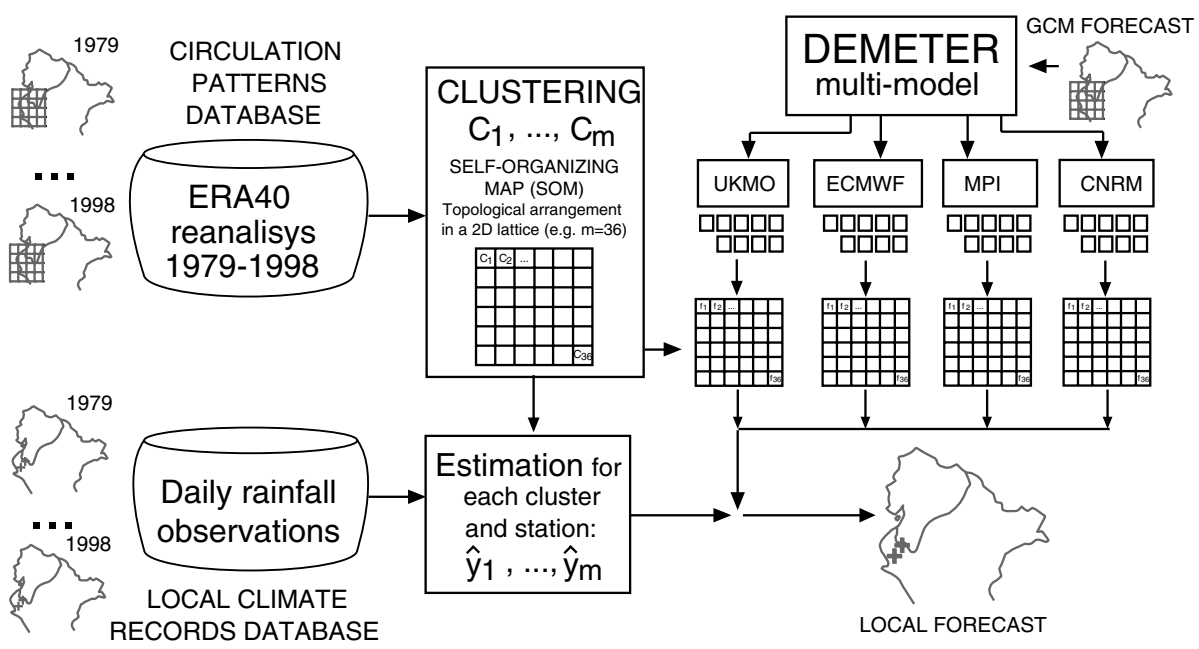

Fig. 10. Schematic diagram of the clustering-based downscaling method. The nine daily members produced for three months (a season) define a PDF on the SOM lattice. The local forecast is finally obtained combining these PDFs with the function assigning the mean precipitation to each cluster and each of the stations. 

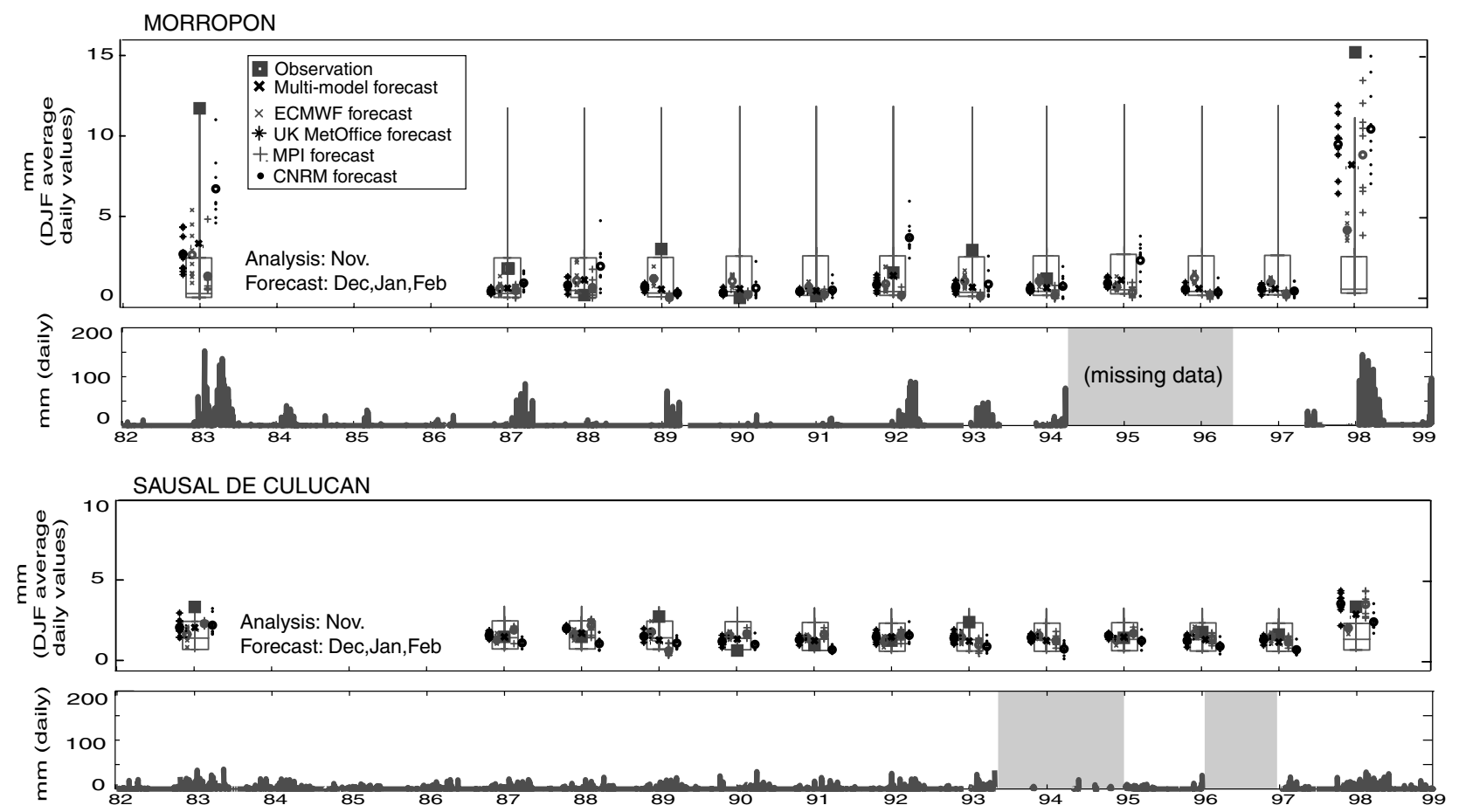

Fig. 11. Box-and-whisker plots of the nine ensemble members of the four models applied for downscaling DJF accumulated seasonal precipitation using 1-4 month lead time seasonal forecasts in two stations for the period 1982-1998, including the two strong El Niño episodes of 1982/1983 and 1997/1998 (the box represents the quartiles of the climatology; whiskers are located at percentiles 5 and 95). Mean daily values are shown in the figure.

\subsection{Seasonal downscaling}

Figure 11 shows the downscaling results for the two stations in Peru for the period 1982-1998. The box-and-whisker graphs shown in this figure correspond to the quartiles of the daily precipitation observations in each of the stations (the vertical lines mark the 5 and 95 percentiles of the observation values). Therefore, observed values above/below the box can be associated with positive/negative anomalies of the precipitation. In this figure, the forecasts for each model and for each ensemble member are shown separately. Thus, for each year, $9 \times 4=36$ numerical forecasts are shown. Moreover, the mean of the ensemble members is identified for each model (labelled with a circle) and the mean of the models is considered as the multimodel forecast (labelled with a cross). This figure shows the forecasts for the period DJF with 1-3 month lead times (November analysis). In both stations, the models skilfully predict the strong positive precipitation anomalies during the strong El Niño events of 1982/1983 and 1997/1998. In particular, the downscaled precipitation is more accurate in the later case. This is not surprising, because the skill of downscaling methods relies on the skill of numerical seasonal models, and they achieved good predictions for El Niño 1997/1998 (Anderson et al., 2003). Overall, the best performance is obtained with the multi-model forecast.
Table 1. Summary of model accumulated seasonal precipitation $(\mathrm{mm})$ forecast for different DJF seasons within the period 1979-1998

\begin{tabular}{lcccc}
\hline Year & CNRM & ECMWF & MPI & UKMO \\
\hline $1979 / 1980$ & 609.4 & 1938.9 & 398.6 & 761.9 \\
$1982 / 1983$ & 557.6 & 2424.7 & 473.9 & 740.2 \\
$1984 / 1985$ & 598.3 & 2952.2 & 207.1 & 747.9 \\
$1997 / 1998$ & 544.0 & 2905.1 & 621.7 & 771.7 \\
\hline
\end{tabular}

To illustrate the performance of this downscaling process, compared with the direct model outputs, we have extracted the seasonal precipitation values forecast by the different DEMETER models in the grid point closest to the two stations (see Fig. 1). Table 1 shows the DJF seasonal accumulated amounts. Note that the numerical model cannot reproduce the different precipitation regimes simultaneously. The ECMWF model exhibits a large positive bias whereas the remaining models show a low variability between normal and anomalous years. The MPI model is the only one providing larger precipitation amounts for the two El Niño years. However, the precipitation amount on the grid is between the real seasonal precipitation observed during DJF at Morropón (e.g. $1300 \mathrm{~mm}$ for 1997/1998) and that observed at Sausal de Culucán (e.g. 360 mm for 1997/1998). 

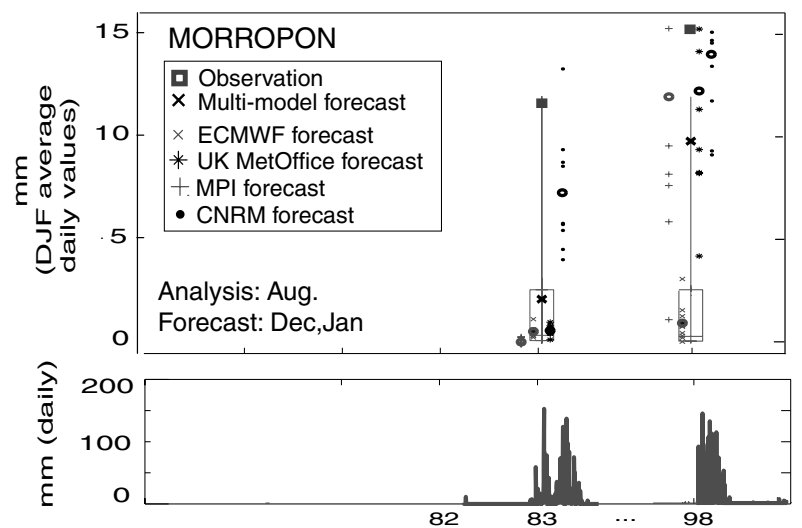

Fig. 12. Box-and-whisker plots of the downscaling accumulated seasonal precipitation of the period December-January (DJ) in two stations for 1982/1983 and 1997/1998 using 5-6 month lead times (August analysis).

Note that the downscaling method presented in this paper (see Fig. 11) provides a much more accurate forecast at both stations, discriminating the different precipitation regimes.

In order to check the skill of the forecast for different lead times, we have also used the August analysis of DEMETER models to compute the 5-6 month lead time local forecast for the period December-January (note that there is no forecast for February for this analysis time). Figure 12 shows the results for the first station (Morropón). In this case, the prediction is still skilful for 1997/1998 but not for 1982/1983 (only one model detects the positive anomaly).

The above downscaling technique can also be used in probabilistic form, computing the probability of exceeding a given threshold using the probabilities defined in the clusters by the observations (see Gutiérrez et al., 2004, for more details). A standard verification methodology for probabilistic forecasting is relative operating characteristics (ROCs). The derivation of ROCs is based on contingency tables for the number of observed occurrences and non-occurrences of an event as a function of the forecast occurrences and non-occurrences (Jolliffe and Stephenson, 2003). Terciles and quartiles of the climatology are standard thresholds used in seasonal forecasts to define wet/normal/dry periods. However, in some stations these thresholds are not appropriate because they do not allow us to discriminate some phenomena of interest. This is the case for Morropón, where even the upper quartile does not discriminate strong from moderate El Niño periods (see the box-and-whisker graphs in Fig. 11). In this case, the threshold is of great importance, and the results may strongly depend on this choice. For instance, Fig. 13 shows the ROC curves obtained for the models and the multi-model for the period 1982-1998 choosing as threshold the percentiles: (a) 80 and (b) 90 . In this last case, there are only two occurrences of the event, corresponding to the strong El Niño periods of $1982 / 1983$ and $1997 / 1998$, where the models have
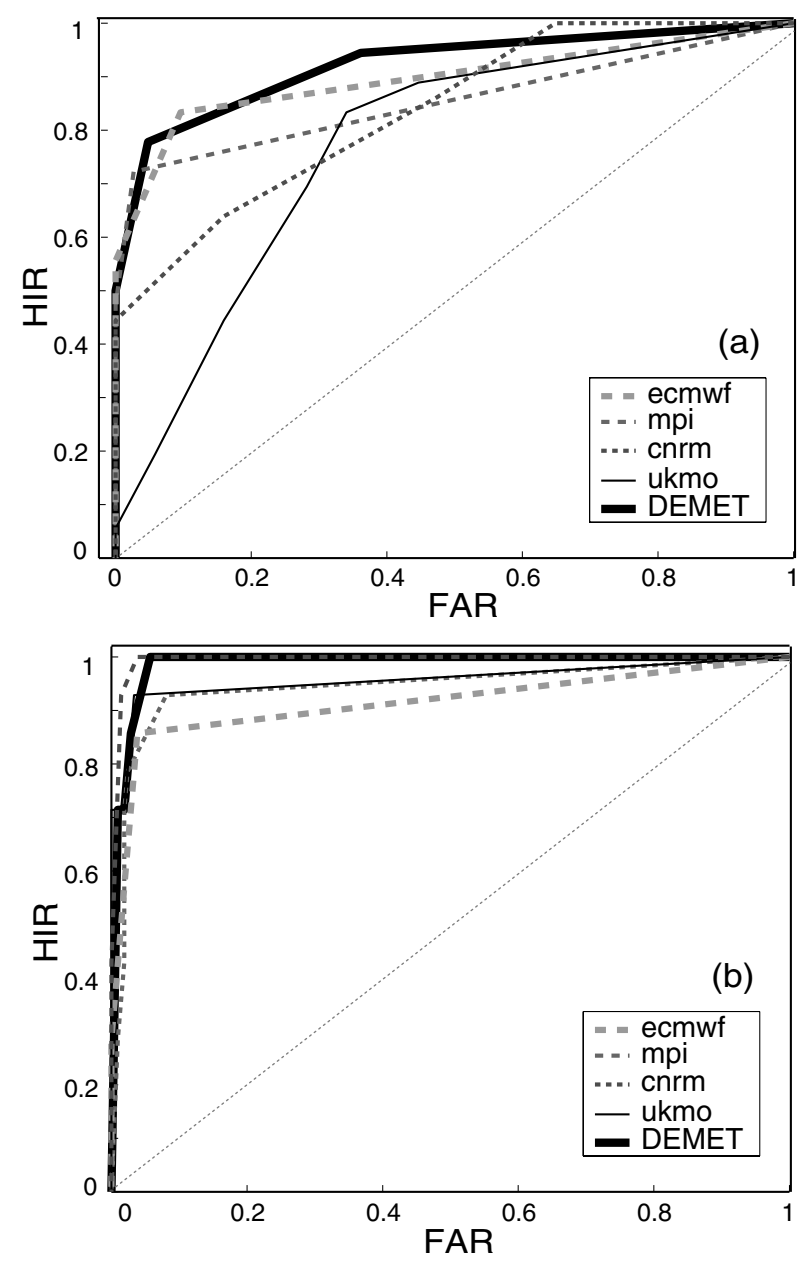

Fig. 13. ROC areas for four models and for the combined multi-model DEMETER for the period DJF 1982-1998 using as a threshold value: (a) percentile 80; (b) percentile 90.

high skill. Again, the best and most robust results correspond to the multi-model ensemble.

\section{Conclusions and further remarks}

In this paper we have illustrated the use of SOMs for analysing and downscaling the seasonal forecasts produced by multi-model ensemble systems, in particular the application to the DEMETER project. We have presented interesting applications of the SOMs for analysing ensembles and comparing the ensembles of different models, extending the work done by Eckert et al. (1996). We have also introduced a simple downscaling method based on the SOMs, extending some ideas previously used in Cavazos (1999, 2000). The skill of both numeric and probabilistic local forecasts is analysed considering two nearby stations in Northern Peru with different precipitation regimes. In conclusion, the multi-model predictions are the most robust and skilful. We show that heavy precipitations associated with strong 
El Niño events can be predicted some months in advance. These results open future work on the application of seasonal forecasts in different sectors, such as agriculture, health and disaster prevention.

\section{Acknowledgments}

The authors are also grateful to the Comisión Interministerial de Ciencia y Tecnología (CICYT, CGL2004-02652 grant) for partial support of this work. We also acknowledge T. Palmer and F. Doblas-Reyes, from the ECMWF, for helpful suggestions for this work. We also acknowledge the anonymous referees for their comments and suggestions, which helped us to improve the paper.

\section{References}

Allan, R. J., Nicholls, N., Jones, P. D. and Butterworth, I. J. 1991. A further extension of the Tahiti-Darwin SOI, early SOI results and Darwin pressure. J. Climate 4, 743-749.

Anderson, D. L. T., Stockdale, T., Balmaseda, M. A., Ferranti, L., Vitart, F. and co-authors. 2003. Comparison of the ECMWF seasonal forecast systems 1 and 2, including the relative performance for the 1997/8 El Niño. Technical Memoranda no. 404, ECMWF.

Cavazos, T. 1999. Large-scale circulation anomalies conductive to extreme precipitation events and derivation of daily rainfall in northeastern Mexico and south-eastern Texas. J. Climate 12, 1506-1523.

Cavazos, T. 2000. Using self-organizing maps to investigate extreme climate event: an application to wintertime precipitation in the Balkans. J. Climate 13, 1718-1732.

Cofiño, A. S., Gutiérrez, J. M., Jakubiak, B. and Melonek, M. 2003. Implementation of data mining techniques for meteorological applications. In Realizing Teracomputing (eds W. Zwieflhofer, and N. Kreitz). World Scientific, Singapore, 215-240.
Díez, E., Primo, C., García-Moya, J. A., Gutiérrez, J. M. and Orfila, B. 2005. Statistical and dynamical downscaling of precipitation over Spain from DEMETER seasonal forecasts. Tellus 57A, 409-423.

Eckert, P., Cattani, A. and Ambühl, J. 1996. Classification of ensemble forecasts by means of an artificial neural network. Meteorol. Appl. 3, 169-178.

Feddersen, H. and Andersen, U. 2005. A method for statistical downscaling of seasonal ensemble predictions. Tellus 57A, 398-408.

Gutiérrez, J. M., Cano, R., Cofiño, A. S. and Rodríguez, M. A. 2004. Clustering methods for statistical downscaling in short-range weather forecast. Mon. Wea. Rev. 132, 2169-2183.

Han, J. and Kamber, M. 2000. Data Mining: Concepts and Techniques. Morgan Kaufmann, San Mateo, CA, pp. 500.

Hewitson, B. C. and Crane, R. G. 2002. Self-organizing maps: applications to synoptic climatology. Climate Res. 22, 13-26.

Jolliffe, I. T. and Stephenson, D. B. 2003. Forecast Verification: A Practitioner's Guide in Atmospheric Science. Wiley, New York.

Kohonen, T. 1995. Self-Organizing Maps. No. 30 in Springer Series in Information Sciences. Springer-Verlag, Berlin, pp. 521.

Oja, E. and Kaski, S. eds. 1999. Kohonen Maps. Elsevier, Amsterdam, pp. 400.

Palmer, T. N., Alessandri, A., Andersen, U., Cantelaube, P., Davey, M. and co-authors. 2004. Development of a European multi-model ensemble system for seasonal to interannual prediction (DEMETER). Bull. Am. Meteorol. Soc. 85, 853-872.

Peña, J. M., Lozano, J. A. and Larrañaga, P. 1999. An empirical comparison of four initialization methods for the k-means algorithm. Pattern Recognition Letters 20, 1027-1040.

Shannon, C. E. 1948. A mathematical theory of communication. Bell Syst. Tech. J. 27, 623-656.

Wilby, R. L. and Wigley, T. M. L. 1997. Downscaling general circulation model output: a review of methods and limitations. Prog. Phys. Geog. 21, 530-548.

Zorita, E. and von Storch, H. 1999. The analog method as a simple statistical downscaling technique: comparison with more complicated methods. J. Climate 12, 2474-2489. 